Urol. int. 1965;19:229

\title{
Conclusions
}

R.G. Bunge

\section{lowa City}

Author's address: Prof. Raymond G. Bunge, Dept. of Urology, University of lowa, lowa City, lowa (USA)

This symposium on intersexuality has, indeed, covered in considerable depth a very interesting and intriguing medical subject. We have been most fortunate to hear the expositions of the foremost scholars in this area and I, for one, have immensely enjoyed the most congenial "Italian atmosphere" and the impromptu discussion of the panelists to whom I extend deepest thanks. Intersexuality has been of interest to medicine for many years and in the recent ones there has been a quickening of interest due mainly, I presume, to the description of the chromatin test, new techniques for identification of chromosomes and the awareness of clinicians that hitherto unsuspected patients with the disorder have been discovered and described.

We have listened to papers dealing with classification of inter-sexes and their diagnosis, the management of such cases, psychological problems evolving from such states; the obstetrical perpelexies of intersexuality in the new born, the surgical reversal of the female adrenal inter sex and the special aspects of the true inter sexes. Included in the discussion of the panelists was the legal problem of this medical disorder.

Again

I would like to thank our host

Professor Sorrentino and his staff for the kind invitation to participate in this symposium and to the panelists: well done. 